\title{
The maser mechanism for solar millisecond spike generation in inhomogeneous plasma
}

\author{
V. G. Vlasov ${ }^{1}$, A. A. Kuznetsov ${ }^{1}$, and A. T. Altyntsev ${ }^{2}$ \\ 1 Irkutsk State Technical University, Irkutsk, Russia \\ 2 Institute of Solar-Terrestrial Physics, Irkutsk, Russia
}

Received 16 October 2001 / Accepted 5 November 2001

\begin{abstract}
The generation of solar millisecond spikes on the maser cyclotron resonance is studied by taking into account the large-scale monotonic magnetic field inhomogeneity and the small-scale plasma inhomogeneity. It is shown that the presence of these inhomogeneities can have a significant effect on the generation of electromagnetic waves. In this case the structure of the inhomogeneities determines the spectral and temporal parameters of emission. A modeling of the spike dynamic spectrum in the presence of a small-scale inhomogeneity of coronal plasma is performed. It is shown that a simple model of the plasma inhomogeneity (traveling density wave) can account for many of the observed features of spikes.
\end{abstract}

Key words. Sun: flares - Sun: radio radiation - Sun: particle emission - masers

\section{Introduction}

Solar radio and microwave narrowband bursts of millisecond duration, the so-called spikes, have been known for as long as several decades (Benz 1986). Their basic features are: a short duration $(<100 \mathrm{~ms})$ that decreases with an increase of the frequency; a narrow spectral band (a few percent of the frequency); a high brightness temperature (up to $10^{15} \mathrm{~K}$ or higher), which suggests a coherent emission process. Spikes occur in series (clusters) containing up to several thousand simple bursts. Most often the spikes are accompanied by hard X-ray bursts. In view of all these features many researchers have concluded that spikes are closely related to energy release processes in the solar corona and therefore have a high diagnostic potential.

However, the spike generation mechanism remains unclear. Among the various coherent processes most often considered are the maser cyclotron resonance (Melrose \& Dulk 1982) and different variants of the plasma mechanism when plasma waves are transformed into radio emission due to nonlinear processes (Zaitsev \& Stepanov 1983; Willes \& Robinson 1996). In this article we consider the maser mechanism of spike generation, namely the generation of the fundamental harmonic of the extraordinary wave (X1-mode). The emission frequency in this case virtually coincides with the electron cyclotron frequency in the source, which requires the presence of sufficiently

Send offprint requests to: A. A. Kuznetsov, e-mail: kuzn@math.istu.irk.ru strong magnetic fields in the solar corona $(>100 \mathrm{G}$ for the emission frequency $>300 \mathrm{MHz}$ ). At the present time the existence of such magnetic fields is questionable; nevertheless, the electron cyclotron maser is still commonly considered to be the generation mechanism of solar radio emission (Fleishman \& Melnikov 1998).

Most publications that have considered the maser mechanism of spike generation (Aschwanden 1990; Fleishman \& Melnikov 1998; Fleishman \& Arzner 2000; and other) are devoted mainly to the investigation of the mutual evolution of the electromagnetic wave and the unstable electron distribution. It is assumed that the main factor limiting the emission intensity is the quasilinear (or nonlinear) relaxation of this unstable distribution. Such a relaxation is very fast and therefore the generation of the emission takes place virtually in the area of formation of the unstable particle distribution. The influence of the inhomogeneities of the medium (magnetic field and plasma) is considered only when determining the spectral band of emission.

At the same time, as Le Quéau et al. (1985), Zarka et al. (1986), Vlasov (1991a, 1991b) have shown, inhomogeneities of the magnetic field and plasma essentially determine the characteristics of auroral kilometric radiation that is generated in the Earth's magnetosphere on the maser cyclotron resonance. The escape of the wave from resonance with the electron beam in the space of wave vectors proved to be the main factor limiting the emission intensity in this case. The combined influence of the large-scale monotonic magnetic field inhomogeneity 
and the small-scale plasma density inhomogeneity forms a complex dynamic spectrum of the auroral kilometric radiation.

In this paper the theory of the generation of radio emission on the maser cyclotron resonance that has been developed for the emission of the magnetospheres of the Earth and other planets (Vlasov 1991a, 1991b; Vlasov \& Kuznetsov 1997), is used to interpret the solar millisecond spikes. In Sect. 2 of this article the basic equations of the process are given. In Sect. 3 it is shown that the unstable electron beams in the solar corona are stabilized by the large-scale longitudinal magnetic field inhomogeneity, the conditions allowing for the generation of the emission are determined, and the scenario for spike generation in an inhomogeneous medium is proposed. In Sect. 4 we introduce a simple model of a small-scale plasma inhomogeneity in the form of a traveling density wave, calculate the dynamic spectrum of the spike corresponding to this inhomogeneity and compare it with an experimental spike spectrum.

\section{The generation of emission in an inhomogeneous medium}

\subsection{The maser cyclotron resonance}

The equation of maser cyclotron resonance (MCR) of electrons and electromagnetic waves at the fundamental harmonic has the form:

$\dot{\psi}=\omega-k_{\|} v_{\|}-\omega_{B} / \Gamma=0$,

where $\psi$ is the relative phase of waves and particles, $\omega$ is the wave frequency, $\omega_{B}$ is the electron cyclotron frequency, $k_{\|}$and $v_{\|}$are the longitudinal (parallel to the magnetic field) components of the wave vector and particle velocity, respectively, and $\Gamma=\left(1-v^{2} / c^{2}\right)^{-1 / 2}$ is the relativistic factor.

Within the semirelativistic approximation $(v \ll c)$, Eq. (1) in the velocity space of electrons $\left(v_{\|}, v_{\perp}\right)$ represents a circle (or semicircle if we assume $v_{\perp}>0$ ) with the center at the point $v_{\|}=W_{0}, v_{\perp}=0$ and the radius $W$ (Melrose \& Dulk 1982)

$W_{0}=c N_{\|}, \quad W=c \sqrt{N_{\|}^{2}-2 \Delta_{1}}$,

where $N_{\|}=c k_{\|} / \omega$ is the longitudinal component of the refraction index, $\Delta_{1}=\left(\omega-\omega_{B}\right) / \omega$.

\subsection{The model of the electron beam}

It was already noted (Melrose \& Dulk 1982) that the growth rate of the electromagnetic waves on MCR can be positive only when the electron distribution function $f_{b}$ has an area with a positive slope $\partial f_{b} / \partial v_{\perp}>0$. In this paper the electron beam with the following distribution function (Vlasov 1991a) is considered to be the emission source:

$f_{b}(v, \theta)=\frac{\varphi(\theta)}{2 \pi^{3 / 2} v_{b}^{2} \Delta v_{b}} \exp \left[-\left(\frac{v-v_{b}}{\Delta v_{b}}\right)^{2}\right]$,

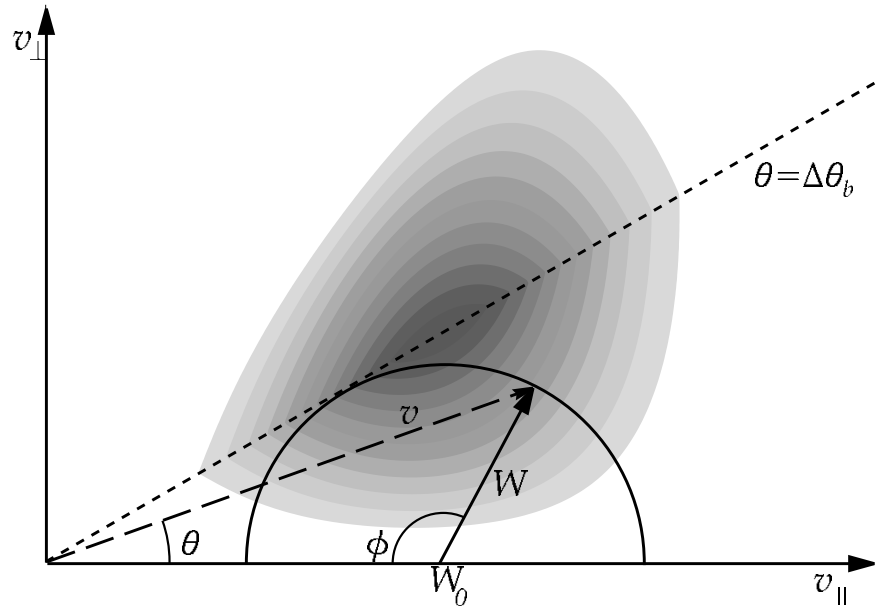

Fig. 1. The model distribution function of the electron beam.

$\varphi(\theta)=\frac{1}{\left(\Delta \theta_{b}\right)^{2}} \begin{cases}\theta / \Delta \theta_{b}, & 0<\theta \leq \Delta \theta_{b}, \\ 2-\theta / \Delta \theta_{b}, & \Delta \theta_{b}<\theta \leq 2 \Delta \theta_{b}, \\ 0, & \theta>2 \Delta \theta_{b},\end{cases}$

where $\theta$ is the electron pitch-angle. This distribution function corresponds to the so-called hollow beam with a maximum at $v=v_{b}, \theta=\Delta \theta_{b}$. Such beams were observed repeatedly in the Earth's magnetosphere where they generate auroral kilometric radiation (Le Quéau et al. 1985; Zarka et al. 1986; Vlasov 1991a, 1991b). In the solar corona, hollow beams can be generated when, for example, particles are reflected from the moving magnetic field gradient ( $\mathrm{Li}$ 1987; Wu et al. 1986). The electron beam (3-4) is shown in Fig. 1.

\subsection{The emission growth rate on $M C R$}

The X1-mode dispersion equation near the cutoff point (when $\omega \simeq \omega_{B}, N_{\|} \ll 1$ ) has the form

$N^{2} \simeq \frac{\Delta_{1}-V}{\Delta_{1}-0.5 V \sin ^{2} \alpha}$

where $V=\left(\omega_{p} / \omega\right)^{2}, \omega_{p}$ is the plasma (Langmuir) frequency, and $\alpha$ is the angle between the wave propagation direction and the magnetic field. The growth rate of the electromagnetic waves on MCR under these conditions reduces to the integral along the resonance semicircle (Vlasov 1991a)

$\gamma_{\mathrm{X} 1} \simeq \frac{n_{b}}{n} \omega \Delta_{1} V(\pi W c)^{2} \int_{0}^{\pi} \frac{\partial f_{b}}{\partial v_{\perp}} \sin ^{2} \phi \mathrm{d} \phi$,

where $n_{b}$ and $n$ are the densities of the electron beam and the background plasma, $n_{b} \ll n$.

For the model electron beam (3-4), the growth rate is positive when $W_{0} \simeq v_{b}$, and the resonance semicircle lies entirely in the area of the positive slope of the distribution function, i.e.

$0<W \leq v_{b} \Delta \theta_{b}$ 
Under this condition the growth rate is

$\gamma_{\mathrm{X} 1} \simeq \frac{n_{b}}{n} \frac{\omega \Delta_{1} V c^{2} W^{2}}{v_{b}^{3} \Delta v_{b}\left(\Delta \theta_{b}\right)^{3}}$

It reaches a maximum at $W=v_{b} \Delta \theta_{b}$.

\subsection{The conditions of X1-mode generation by the hollow beam}

The condition (7) imposes constraints on the plasma and magnetic field parameters, under which the hollow beam (3-4) can generate emission on MCR. First we consider the generation conditions for the wave with the propagation parallel to the magnetic field (when $\alpha=0$ ). If we introduce the parameter $\varepsilon=\left(\omega_{p} / \omega_{B}\right)^{2}$, then, taking into account the dispersion Eq. (5), the characteristics of the resonance semicircle $(2)$, and the relation $W_{0} \simeq v_{b}$, we have

$\varepsilon_{0}>\varepsilon \geq \varepsilon_{0}\left[1-\left(\Delta \theta_{b}\right)^{2}\right]$,

$\varepsilon_{0}=\frac{1}{2} \frac{v_{b}^{2}}{c^{2}}\left(1-\frac{v_{b}^{2}}{c^{2}}\right) \leq \frac{1}{8}$.

Thus the region of the X1-mode generation is limited to a relatively low-density plasma. A maximum growth rate corresponds to the lower boundary of the interval (9). If the magnetic field intensity $B=100 \mathrm{G}$, the electron beam with the energy $E_{b}=20 \mathrm{keV}$ and the pitchangle dispersion $\Delta \theta_{b}=1 / 3$ can generate the X1-mode emission when the plasma density is within the interval $(2.95-3.32) \times 10^{7} \mathrm{~cm}^{-3}$.

Figure 2 shows the regions of parameters, under which electron beams with different energies can generate emission with an arbitrary propagation direction. It is evident from the figure that the interval of possible propagation angles is quite wide. The angle that corresponds to a maximum growth rate varies depending on particular generation conditions, but the value of a maximum growth rate itself remains approximately constant. The sole exception is the case of the wave propagation at large angles to the magnetic field when their amplification is less effective or impossible.

\subsection{The emission intensity}

Within the linear approximation the spectral density of electromagnetic wave energy $W_{f}$ increases exponentially with time, i.e.

$W_{f}=W_{f 0} \exp \left[\left(2 \gamma_{\mathrm{X} 1}-\nu_{e}\right) \Delta t\right]$,

where $W_{f 0}=\Omega \omega^{2} k_{B} T /\left(2 \pi^{2} v_{\mathrm{f}}^{2} v_{\text {gr }}\right)$ is the energy of thermal oscillations $(\Omega$ is the solid angle where the emission is concentrated, $T$ is the plasma temperature, $v_{\mathrm{f}}$ and $v_{\text {gr }}$ are the phase and group wave velocities, respectively, $\nu_{e}$ is the collision frequency), and $\Delta t$ is the time during which the wave is in resonance with the beam.

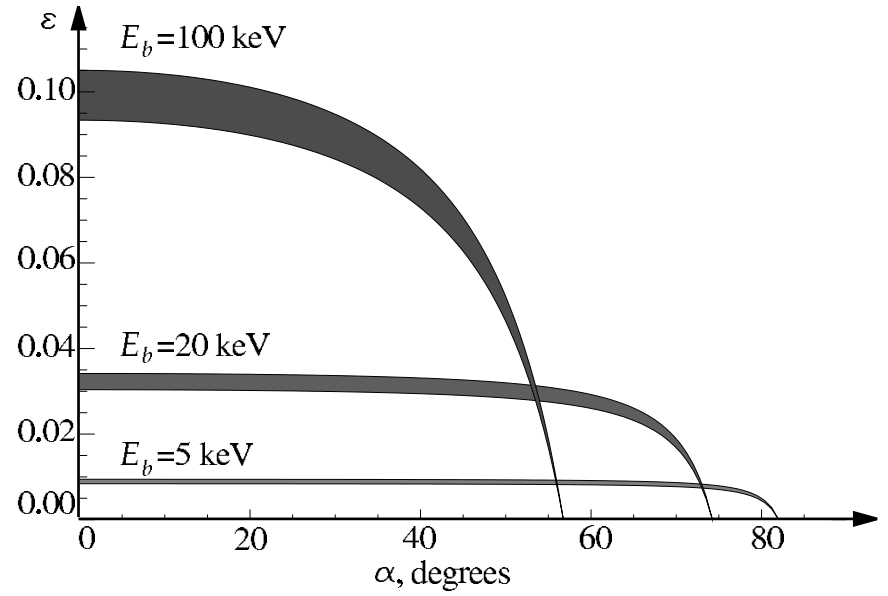

Fig. 2. The parameters, under which the hollow beam can generate emission on MCR. The beam pitch-angle dispersion $\Delta \theta_{b}=1 / 3$.

The spectral density of the emission intensity observed at the Earth (neglecting the absorption during the propagation) can be estimated as

$I_{f} \simeq \frac{W_{f} v_{\mathrm{gr}}}{\Omega}\left(\frac{r_{0}}{R}\right)^{2}$,

where $r_{0}$ is the source size, and $R$ is the distance from the Sun to the Earth.

\subsection{The resonance time}

The time of resonance $\Delta t$ is determined by the escape of the wave from the generation region both in the ordinary space and in the space of wave vectors, i.e. mainly by the breakdown of the phase synchronism condition (1). To determine this value we expand the derivative of the relative phase of waves and particles (1) in the vicinity of the point $t=t_{0}$ into a series at $\Delta t=t-t_{0}$ (Vlasov 1991a):

$\Delta \dot{\psi}=\delta \dot{\psi}+\ddot{\psi}\left(t_{0}\right) \Delta t+\dddot{\psi}\left(t_{0}\right) \frac{(\Delta t)^{2}}{2}+\ldots=0$.

In Eq. (13), $\delta \dot{\psi}$ is the variation of the derivative of the relative phase with respect to electron velocities, which determines the degree of divergence of the particle distribution from a monoenergetic one

$\delta \dot{\psi}=\frac{\partial \dot{\psi}}{\partial v} \Delta v+\frac{\partial \dot{\psi}}{\partial \theta} \Delta \theta \simeq \omega\left(\frac{v_{b} \Delta \theta_{b}}{c}\right)^{2}$

(an approximate value is obtained for the beam $(3-4)$ when $\omega \simeq \omega_{B}$ ).

Instead of the exact solution of Eq. (13), it is possible to use the approximate roots

$\Delta t_{1}=\left|\delta \dot{\psi} / \ddot{\psi}\left(t_{0}\right)\right|, \quad \Delta t_{2}=\sqrt{\left|2 \delta \dot{\psi} / \dddot{\psi}\left(t_{0}\right)\right|}$.

The resonance time is determined by the least of these values, $\Delta t \simeq \min \left(\Delta t_{1}, \Delta t_{2}\right)$. 


\subsection{The propagation of the electromagnetic wave in an inhomogeneous medium}

We consider the model where the magnetic field $B$ is stationary in time and is characterized by constant scales of spatial inhomogeneity $L_{B \|}$ and $L_{B \perp}$

$L_{B \|, \perp}=\frac{B}{\partial B / \partial r_{\|, \perp}}$,

where $r_{\|}$and $r_{\perp}$ are the coordinates along and across the magnetic field line, respectively.

The plasma density is characterized by the scales of spatial inhomogeneity $L_{p \|}$ and $L_{p \perp}$, and by the time of variation $\tau_{p}$

$L_{p \|, \perp}=\frac{n}{\partial n /\left.\partial r_{\|, \perp}\right|_{t}}, \quad \tau_{p}=\frac{n}{\partial n /\left.\partial t\right|_{r}}$

where these parameters can also depend on the coordinates and time.

Under these assumptions the second derivative of the relative phase of waves and particles is

$\ddot{\psi} \simeq-\dot{k}_{\|} v_{\|}-\frac{\omega_{B}}{\Gamma}\left(\frac{\dot{r}_{\|}}{L_{B \|}}+\frac{\dot{r}_{\perp}}{L_{B \perp}}\right)$.

When $\omega \simeq \omega_{B}$, the first term is dominant in the expression (18).

The derivatives $\dot{k}_{\|, \perp}$ and $\dot{r}_{\|, \perp}$ are calculated using the Hamiltonian equations (see Vlasov 1991b) and are

$$
\begin{aligned}
& \dot{k}_{\|, \perp} \simeq-\omega \frac{\delta_{\|, \perp}}{L_{B \|, \perp}} \frac{1-N^{2}}{1-N^{2}+2 \Delta_{1}}-\omega \frac{V N_{\|, \perp}}{4 c \tau_{p}} \frac{2-N_{\perp}^{2}}{\Delta_{1}-V}, \\
& \dot{r}_{\|}=\simeq \frac{2 c N_{\|} \Delta_{1}}{1-N^{2}+2 \Delta_{1}}, \quad \dot{r}_{\perp}=\simeq \frac{2 c N_{\perp} \Delta_{1} \mu_{\perp}}{1-N^{2}+2 \Delta_{1}},
\end{aligned}
$$

where the parameters $\delta_{\|, \perp}=1+\Delta_{1} L_{B \|, \perp} / L_{p \|, \perp}$, and $\mu_{\perp}=\left(1+N_{\|}^{2}\right) /\left(2-N_{\perp}^{2}\right)$ are used.

The third derivative of the relative phase of waves and particles has a more complicated expression. We give here its approximate value for the wave with the propagation parallel to the magnetic field $(\alpha=0)$ :

$$
\begin{aligned}
\dddot{\psi} \simeq & \frac{\omega v_{\|}}{L_{B \|}}\left[2 \delta_{\|}\left(\dot{r}_{\|} \frac{\delta_{\|}+1}{L_{B \|}}+\frac{V}{\tau_{p}}\right)-\frac{L_{B \|}}{L_{p \|}}\left(\frac{\dot{r}_{\|}}{L_{B \|}}\right.\right. \\
& \left.\left.+\Delta_{1} \frac{\dot{L}_{p \|}}{L_{p \|}}\right)\right]+\frac{\omega v_{\|}}{2 c N_{\|} \tau_{p}}\left(\frac{c \delta_{\|}}{N_{\|} L_{B \|}}+\frac{\mu_{\perp}}{N_{\|}^{2} \tau_{p}}-\frac{\dot{\tau}_{p}}{\tau_{p}}\right) \\
& +\frac{\omega_{B} c}{\Gamma N_{\|} L_{B \|}}\left(\frac{\dot{r}_{\|} \delta_{\|} \sigma}{L_{B \|}}+\frac{V}{\tau_{p}}\right)
\end{aligned}
$$

where $\sigma=1+\mu_{\perp}\left(\delta_{\perp} / \delta_{\|}\right)\left(L_{B \|}^{2} / L_{B \perp}^{2}\right)$. The derivatives $\dot{L}_{p \|}$ and $\dot{\tau}_{p}$ include the dependence both on the time and on the coordinates

$$
\begin{aligned}
& \dot{L}_{p \|}=\left.\dot{r}_{\|} \frac{\partial L_{p \|}}{\partial r_{\|}}\right|_{r_{\perp}, t}+\left.\dot{r}_{\perp} \frac{\partial L_{p \|}}{\partial r_{\perp}}\right|_{r_{\|}, t}+\left.\frac{\partial L_{p \|}}{\partial t}\right|_{r}, \\
& \dot{\tau}_{p}=\left.\dot{r}_{\|} \frac{\partial \tau_{p}}{\partial r_{\|}}\right|_{r_{\perp}, t}+\left.\dot{r}_{\perp} \frac{\partial \tau_{p}}{\partial r_{\perp}}\right|_{r_{\|}, t}+\left.\frac{\partial \tau_{p}}{\partial t}\right|_{r}
\end{aligned}
$$

\subsection{The spectral bandwidth of emission}

The frequency range of the emission generated on MCR $\Delta \omega$ depends mainly on two factors. Firstly, it is the limited spatial size of the generation region $\Delta r_{\|, \perp}$. The corresponding spectral bandwidth is

$\frac{\Delta \omega}{\omega} \simeq \max \left(\frac{\Delta r_{\|}}{L_{B \|}}, \frac{\Delta r_{\perp}}{L_{B \perp}}\right)$.

Secondly, even in a homogeneous medium the nonmonoenergetic electron beam amplifies electromagnetic waves with different frequencies. To estimate the corresponding frequency range, we write the variation of the derivative of the relative phase of waves and particles (1) with respect to the frequency and to the electron velocities:

$\Delta \dot{\psi}=\frac{\partial \dot{\psi}}{\partial \omega} \Delta \omega+\frac{\partial \dot{\psi}}{\partial v} \Delta v+\frac{\partial \dot{\psi}}{\partial \theta} \Delta \theta=0$.

For the hollow beam (3-4) we obtain

$\frac{\Delta \omega}{\omega} \simeq\left(\frac{v_{b} \Delta \theta_{b}}{c}\right)^{2}$.

In most cases the nonmonoenergetic distribution of the electron beam has a dominant influence on the spectral bandwidth of spikes and emission of planetary magnetospheres.

\subsection{The collective relaxation of the electron beam}

The energy flux of the electron beam that is transferred through a unit area per unit time is

$\Phi=E_{b} n_{b} v_{b}$.

On the other hand, per unit time per unit volume the energy transferred from the beam to electromagnetic waves is

$$
\frac{\partial \Phi}{\partial r} \simeq\left(2 \gamma_{\mathrm{X} 1}-\nu_{e}\right) W_{f} \Delta f
$$

(here it is assumed that the growth rate and the spectral density of the emission energy are nearly constant within the spectral band $\Delta f$ ). This value determines the rate of beam energy losses. We define the length of collective relaxation as the distance where the electron beam loses $10 \%$ of its total energy. The electron distribution function may be considered unchanged within distances which are much shorter than the length of collective relaxation.

\section{The stabilization conditions for electron beams in the solar corona}

\subsection{The stabilization of electron beams}

As shown in the previous section, the duration of the resonance, and hence the intensity of the radio emission generated on MCR depend strongly on the scales and dynamics of magnetic field and plasma inhomogeneities. First 
we consider the case where the plasma density is timeindependent and is characterized by constant scales of spatial inhomogeneity $L_{p \|}$ and $L_{p \perp}$ (i.e. it is assumed that $\tau_{p}=\infty$, and $\left.\dot{L}_{p \|}=\dot{L}_{p \perp}=0\right)$. The main factors that influence the resonance time are the scales of longitudinal inhomogeneity $L_{B \|}$ and $L_{p \|}$.

Tables 1-2 give an example of a calculation of the generation of radio emission on MCR. The electromagnetic wave propagation direction is parallel to the magnetic field. Table 1 presents the parameters that do not depend on the longitudinal inhomogeneity scale of plasma density. The chosen scales of magnetic field inhomogeneity are on the order of the coronal magnetic loop dimensions. The parameters of the electron beam distribution function are similar to electron beams observed in the Earth's magnetosphere.

If the scale of longitudinal inhomogeneity of plasma density $L_{p \|} \rightarrow \infty$ (or is comparable with the scale of longitudinal inhomogeneity of the magnetic field, see Table 2), then the resonance time is determined by the time $\Delta t_{1}$, which, in turn, is proportional to the scale of longitudinal inhomogeneity of the magnetic field:

$\left.\Delta t_{1}\right|_{L_{p \|}=\infty} \simeq \frac{L_{B \|}}{c} \frac{v_{b}}{c}\left(\Delta \theta_{b}\right)^{2}$.

Under conditions typical of the solar corona the time $\Delta t_{1}$ is short; therefore, the electron beam with the parameters shown in Table 1 cannot amplify the electromagnetic waves to a considerable level despite a rather high growth rate. As a consequence, the beam energy losses due to emission are small, and the possible propagation distance of the beam far exceeds the size of the coronal magnetic loop. Conceivably a large-scale longitudinal inhomogeneity of the magnetic field in the solar corona stabilizes unstable electron beams (if their intensity does not exceed a certain level), thus allowing them to propagate to large distances from the formation region.

\subsection{The compensation of plasma and magnetic field inhomogeneities}

We now consider the dependence of the resonance time on the scale of longitudinal inhomogeneity of plasma. At the certain ratio of the scales of longitudinal inhomogeneities of plasma and magnetic field, namely at

$L_{p \|} \simeq-\Delta_{1} L_{B \|}$

the second derivative of the relative phase of waves and particles $\ddot{\psi}$ goes to zero. In this case the time $\Delta t_{1}$ goes to infinity and the resonance time is determined by the time $\Delta t_{2}$, which under the condition (28) is

$\left.\Delta t_{2}\right|_{L_{p \|} \simeq-\Delta_{1} L_{B \|}} \simeq \frac{L_{B \|}}{c} \Delta \theta_{b}\left(1+\frac{\delta_{\perp} \mu_{\perp}}{2} \frac{L_{B \|}^{2}}{L_{B \perp}^{2}}\right)^{-1 / 2}$.

Thus the compensation of the longitudinal inhomogeneities of plasma and magnetic field (28) results in a

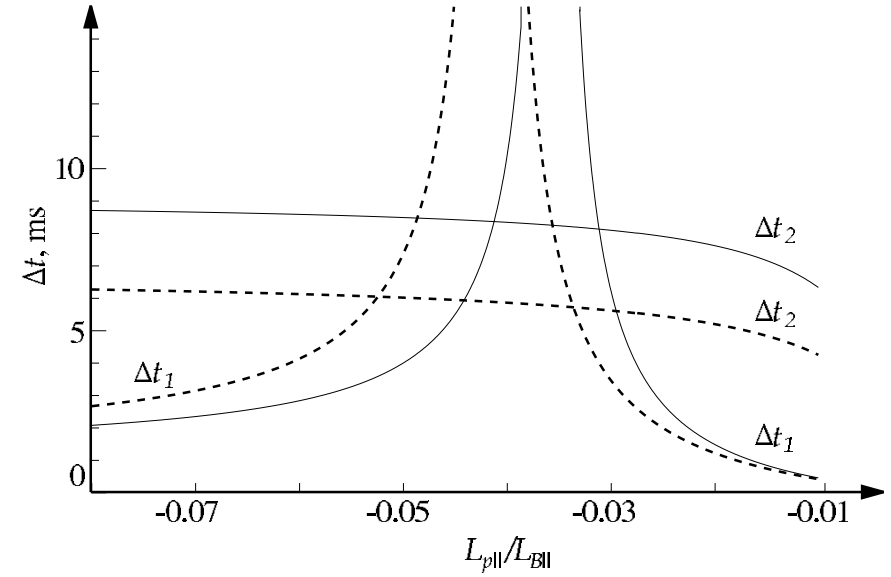

Fig. 3. The dependence of the resonance times on the scale of longitudinal inhomogeneity of plasma density. The solid and dashed lines correspond to the wave propagation directions $\alpha=$ 0 and $\alpha=45^{\circ}$, respectively.

significant increase of the resonance time. As can be seen from Table 2, under the condition of compensation of the longitudinal inhomogeneities the energy transfer from the electron beam to the electromagnetic waves becomes far more effective, the stabilization fails, and the emission intensity increases many times.

Figure 3 shows the dependence of the resonance times $\Delta t_{1}$ and $\Delta t_{2}$ on the scale of longitudinal inhomogeneity of plasma for different propagation directions of the wave. The other parameters of the magnetic field, plasma and electron beam are the same as in Table 1. An increase of the resonance time due to the compensation of the longitudinal inhomogeneities occurs at any angle between the wave propagation direction and the magnetic field, but the exact condition of this compensation can somewhat change. The resonance time $\Delta t_{2}$ decreases with an increase of the angle between the wave propagation direction and the magnetic field, which makes the quasilongitudinal propagation somewhat more favorable.

The resonance time $\Delta t_{1}$ (and therefore the conditions of electron beam stabilization) does almost not depend on the transverse inhomogeneities of the magnetic field and plasma. However, as follows from the expression (29), these inhomogeneities have a significant influence on the resonance time $\Delta t_{2}$. A detailed investigation of the effects associated with the influence of the transverse inhomogeneities of the magnetic field and plasma on the generation of emission on MCR, is beyond the scope of this paper.

\subsection{Dynamic spectrum of radio emission as the reflection of the structure of plasma inhomogeneities}

As the expression (28) suggests, for the breakdown of the electron beam stabilization the required scale of the longitudinal inhomogeneity of plasma density must be, firstly, much smaller than the scale of longitudinal inhomogeneity 
Table 1. The parameters of the magnetic field, plasma and electron beam.

\begin{tabular}{lll}
\hline Electron cyclotron frequency & $f_{B}, \mathrm{MHz}$ & 350 \\
Magnetic field inhomogeneity scales & $L_{B \|}, \mathrm{km}$ & 10000 \\
Plasma density & $L_{B \perp}, \mathrm{km}$ & 5000 \\
Plasma frequency & $n, \mathrm{~cm}^{-3}$ & $5 \times 10^{7}$ \\
Plasma temperature & $f_{p}, f_{B}$ & 0.181 \\
Transverse scale of plasma density inhomogeneity & $T, \mathrm{~K}$ & $10^{6}$ \\
Thermal oscillation energy density at $f \simeq f_{B}, \Omega=\pi / 2$ & $L_{p \perp}, \mathrm{km}$ & 5000 \\
Energy of the electron beam & $W_{f 0}, \mathrm{erg} \mathrm{cm}^{-3} \mathrm{~Hz}^{-1}$ & $1.57 \times 10^{-21}$ \\
Velocity of the electron beam & $E_{b}, \mathrm{keV}$ & 20 \\
Density of the electron beam & $v_{b}, \mathrm{c}$ & 0.272 \\
Velocity dispersion of the electron beam & $n_{b}, n$ & $4.4 \times 10^{-6}$ \\
Pitch-angle dispersion of the electron beam & $\Delta v_{b}, v_{b}$ & $1 / 3$ \\
Growth rate of electromagnetic waves & $\Delta \theta_{b}$ & $1 / 3$ \\
Collision frequency & $\gamma_{\mathrm{X} 1}, \mathrm{~Hz}$ & $1.12 \times 10^{3}$ \\
Minimum spectral bandwidth of the emission & $\nu_{e}, \mathrm{~Hz}$ & 3.90 \\
Energy flux of the electron beam & $\Delta f, \mathrm{MHz}$ & 2.97 \\
\hline
\end{tabular}

Table 2. The characteristics of the emission generation process at different scales of longitudinal inhomogeneity of plasma density.

\begin{tabular}{llll}
\hline Scale of longitudinal inhomogeneity of plasma density & $L_{p \|}, \mathrm{km}$ & 10000 & -356 \\
Resonance times & $\Delta t_{1}, \mathrm{~ms}$ & 1.12 & $\infty$ \\
Emission energy density & $\Delta t_{2}, \mathrm{~ms}$ & 9.09 & 8.26 \\
Emission intensity at the Earth * & $W_{f}, \mathrm{erg} \mathrm{cm}^{-3} \mathrm{~Hz}^{-1}$ & $1.90 \times 10^{-20}$ & $1.64 \times 10^{-13}$ \\
Energy loss rate of the electron beam & $I_{f}, \mathrm{sfu}$ & $2.93 \times 10^{-5}$ & 252 \\
Collective relaxation length of the electron beam & $\partial \Phi / \partial r, \mathrm{erg} \mathrm{cm}^{-3} \mathrm{~s}^{-1}$ & $1.26 \times 10^{-10}$ & $1.09 \times 10^{-3}$ \\
\hline
\end{tabular}

* The source size is $r_{0} \simeq 100 \mathrm{~km}$, and the absorption is neglected.

of the magnetic field and, secondly, the longitudinal components of plasma and magnetic field gradients must be oppositely directed. This is possible only due to a smallscale plasma inhomogeneity (turbulence). Accordingly, a breakdown of the stabilization can take place only in relatively narrow height ranges, which leads to a narrowband spectrum of the generated emission. Note that if the spatial dimensions of the generation region are determined only by the condition of compensation of the inhomogeneities (28), then the spatial escape of the electromagnetic waves from the generation region is already taken into account in the resonance time $\Delta t_{2}$ in view of the relations (21).

Thus we can propose the following scenario for generation of the spikes on MCR in the inhomogeneous medium. The region of formation of an unstable electron beam and the region of generation of emission are spatially separated. The large-scale longitudinal inhomogeneity of the magnetic field stabilizes the beam both in the formation region and during the propagation. The lifetime of the beam far exceeds the duration of a single spike and corresponds to the duration of a series of spikes. The generation of emission takes place only in those local regions where the small-scale inhomogeneity of plasma density slows down the escape of the electromagnetic waves from resonance with the beam and thus breaks the stabilization. The emission spectrum at any instant of time is the picture of the structure of the plasma small-scale inhomogeneity. The duration of a single spike is determined by the lifetime of the corresponding inhomogeneity. The spatial movement of the inhomogeneity results in a shift of the generation region and in a frequency drift of the emission. The time variation of the inhomogeneity forms the time profile of emission intensity. The transverse size of the local generation region is determined by the smallscale plasma inhomogeneity in the same way as does the longitudinal size and is most likely to be much smaller $\left(\Delta r_{\perp}\right.$ is of the same order of magnitude as $\left.L_{p \|}\right)$ than the transverse size of the coronal magnetic loop and the diameter of the electron beam.

This scenario is supported by X-ray observations. As Güdel et al. (1991) pointed out, X-ray bursts correlate not with single spikes but with series of spikes, i.e. the duration of the X-ray burst is comparable with that of series of spikes and the spike occurrence rate in many cases is virtually proportional to the X-ray intensity. These features are easily explained if both the duration of the X-ray burst and the duration of series of spikes are determined by the lifetime of the electron beam. A change of the electron beam cross-section results in a proportional change 
of the X-ray intensity and, at the same time, in a proportional change of the number of local regions of generation of radio emission (if it is assumed that the plasma density inhomogeneities required for the generation of spikes arise with equal probability in different regions of the coronal magnetic loop).

Almost all spikes are accompanied by X-ray emission. At the same time, only about $2 \%$ of X-ray bursts are accompanied by spikes (Güdel et al. 1991). In the rest of cases either the electron beam distribution function is stable with respect to MCR or the nesessary (for the generation of radio emission) plasma inhomogeneity is missing.

\section{Modeling of the dynamic spectrum of spikes}

\subsection{The model of plasma inhomogeneity}

Here we consider in more detail the generation of radio emission on MCR in the presence of a small-scale plasma inhomogeneity. Assume that the electromagnetic wave propagation direction is parallel to the magnetic field. The physical conditions in the generation region depend only on the longitudinal coordinate (height) $r_{\|}$, which corresponds to a small transverse size of the generation region $\left(r_{\perp} \ll L_{B \perp}, L_{p \perp}\right)$. The magnetic field decreases exponentially with height, $B=B_{0} \exp \left(-r_{\|} / L_{B \|}\right)$, and the electron beam density is proportional to the magnetic field intensity as particles move along the field lines.

The height dependence of the plasma density includes large-scale and small-scale inhomogeneities. The largescale inhomogeneity is a global decrease in plasma density with height with a typical scale $L_{p 0}$. As has been noted above, the breakdown of electron beam stabilization and the generation of emission require the presence of a small-scale plasma inhomogeneity with a sufficiently sharp gradient that is directed oppositely to the magnetic field gradient. Hence in some local region the plasma density must increase with height. In addition, the interpretation of the emission frequency drift requires the presence of a traveling inhomogeneity. We use the following model dependence of the plasma density on the height which satisfies the above mentioned requirements:

$n=n_{0} \exp \left(-\frac{r_{\|}}{L_{p 0}}\right)\left[1+a z \exp \left(-z^{2}\right)\right]$,

$z=\frac{r_{\|}-u t}{d}$.

This expression corresponds to the "plasma density wave" moving with the velocity $u$ which is superimposed on the large-scale inhomogeneity. The profile of the inhomogeneity wave (30) is shown in Fig. 4. The parameters $a$ and $d$ are determined by the amplitude $\Delta n / n$ and size $D$ of the inhomogeneity wave, respectively:

$a=\sqrt{2 e} \frac{\Delta n}{n}, \quad d=\frac{D}{\sqrt{2}}$.

Note that we do not make any assumptions about the origin of the small-scale plasma inhomogeneity. When choosing the functional dependence (30), we sought to find the

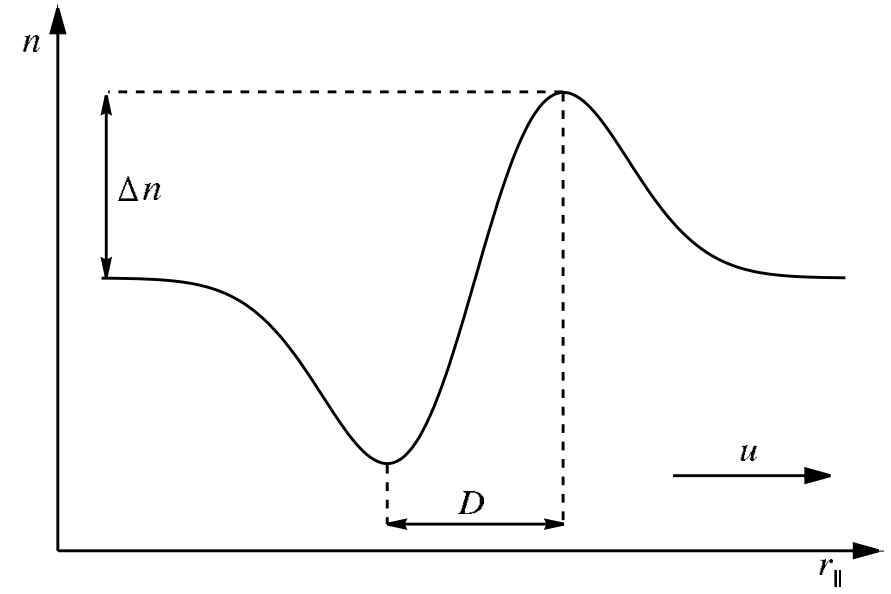

Fig. 4. The profile of a model small-scale inhomogeneity of plasma density.

simplest expression that would allow the agreement of the calculated parameters of radio emission with the experimental ones to be obtained.

The condition of compensation of the longitudinal inhomogeneities of plasma and magnetic field (28) in a non-stationary medium that is described by Eq. (30), somewhat changes and takes the form:

$L_{p \|} \simeq-\Delta_{1} L_{B \|}\left(1-\frac{V}{\Delta_{1}} \frac{u}{\dot{r}_{\|}}\right)$.

\subsection{Comparison of calculated and observed parameters of spikes}

As has been pointed out above, the lifetime of the electron beam far exceeds the spike duration. Therefore when calculating the emission parameters the beam can be considered stationary. By varying the parameters of the electron beam (3-4) and the small-scale plasma inhomogeneity (30), we obtain different dynamic spectra of radio emission, i.e. the dependence of the emission intensity on the frequency and time. Figures 5-7 give an example of such a dependence. The same parameters of the magnetic field, plasma and electron beam as given in Table 1 were used, with the only difference that most of them now depend on the coordinate $r_{\|}$, so instead of "plasma density" there should be "plasma density at the point where $f_{B}=350 \mathrm{MHz}$ ", etc. The scale of large-scale longitudinal inhomogeneity of plasma density affects only slightly the local process of generation of emission. It was taken to be $L_{p 0}=10000 \mathrm{~km}$. The wave of the small-scale plasma inhomogeneity had the following parameters: the amplitude $\Delta n / n=0.2$, the size $D=150 \mathrm{~km}$, and the velocity $u=1000 \mathrm{~km} \mathrm{~s}^{-1}$ (the wave goes upwards). The beam energy losses due to emission are small, so we can use a linear approximation.

Figure 5 shows the calculated two-dimensional dynamic spectrum of radio emission. The movement of the inhomogeneity results in a frequency drift of the emission with a rate of about $38 \mathrm{MHz} \mathrm{s}^{-1}$. The change of the 


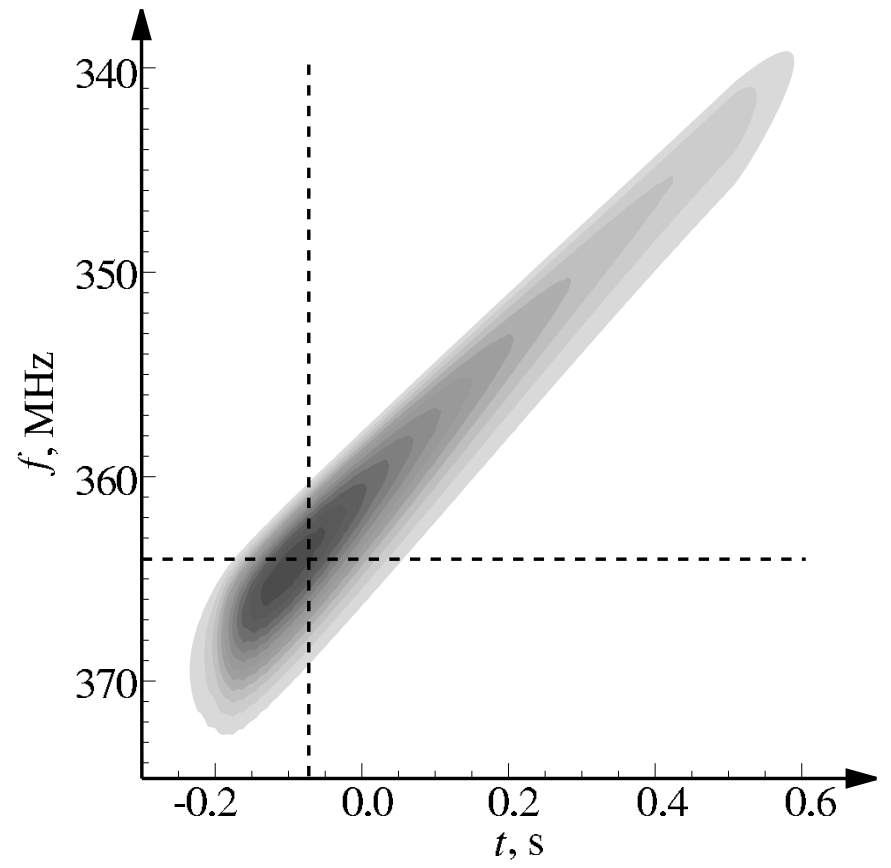

Fig. 5. The calculated dynamic spectrum of radio emission. At the time $t=0 \mathrm{~s}$ the small-scale plasma inhomogeneity wave is centered on the point where the electron cyclotron frequency $f_{B}=350 \mathrm{MHz}$

emission intensity along the inhomogeneity wave trajectory is caused by a change of the growth rate of the electromagnetic waves. A total frequency range, over which the emission occurs, is about $32 \mathrm{MHz}$, and is determined by the X1-mode generation condition (9) and depends on the electron beam parameters and the ratio of the large-scale longitudinal inhomogeneities of plasma $L_{p 0}$ and magnetic field $L_{B \|}$.

Figure 6 plots the emission intensity versus frequency at a fixed time. The spectral bandwidth at $1 / e$ level is $6 \mathrm{MHz}$ or $1.65 \%$ of the emission frequency. The main factor that influence the bandwidth is the shape of the electron beam distribution function, see the relation (24). The observed relative bandwidth of spikes (Csillaghy \& Benz 1993; Messmer \& Benz 2000) varies from $4.1 \%$ to $0.17 \%$ (or smaller). Emission with a relative spectral bandwidth of $0.17 \%$ can be generated by a hollow beam with the pitch-angle dispersion $\Delta \theta_{b} \simeq 0.15$ and energy $E_{b}=20 \mathrm{keV}$.

Figure 7 plots the emission intensity at the fixed frequency $f=364 \mathrm{MHz}$ versus time. The burst duration at $1 / e$ level is $154 \mathrm{~ms}$. The noticeable feature is the asymmetric shape of the time profile - it consists of phases of fast rise and nearly exponential decay. Spike observations with high temporal resolution (Güdel \& Benz 1990) show that such a time profile is an essential characteristic of spikes (for comparison, Fig. 8 shows an experimental time profile). In earlier publications the temporal dependence of the spike intensity was related to the collisional damping (Güdel \& Benz 1990) or the saturation of an unstable electron distribution (Aschwanden 1990;

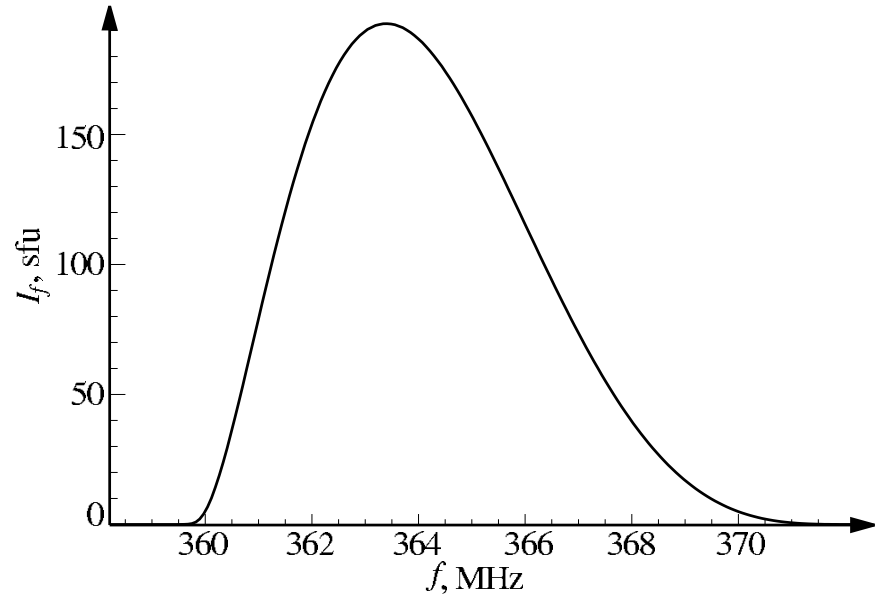

Fig. 6. The calculated spectrum of radio emission (corresponds to the time $t=-0.07 \mathrm{~s}$ in Fig. 5).

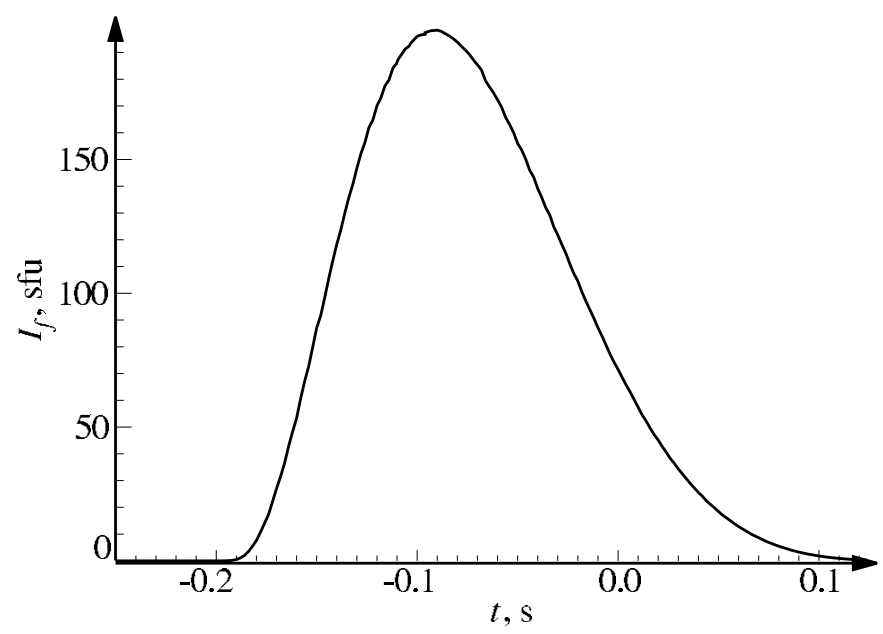

Fig. 7. The calculated time profile of radio emission intensity (emission frequency $f=364 \mathrm{MHz}$ ).

Fleishman \& Arzner 2000). In the case under investigation the time profile is determined by a complex dependence of the resonance time and growth rate on the varying conditions of generation of emission. We are reminded that the time profile shown in Fig. 7 corresponds to the small-scale plasma inhomogeneity wave with the size $D=150 \mathrm{~km}$ moving upwards with the velocity $u=1000 \mathrm{~km} \mathrm{~s}^{-1}$.

\section{Conclusion}

The theory of generation of radio emission on the maser cyclotron resonance in an inhomogeneous medium, developed earlier for radio emission of the planetary magnetospheres, has been used to interpret the solar millisecond spikes. A hollow electron beam was used as the emission source. It has been found that the main factor limiting the amplification of the electromagnetic waves is the escape of the waves from resonance with the beam in the space of wave vectors due to the influence of the inhomogeneities of the magnetic field and plasma density. The longitudinal inhomogeneity of magnetic 


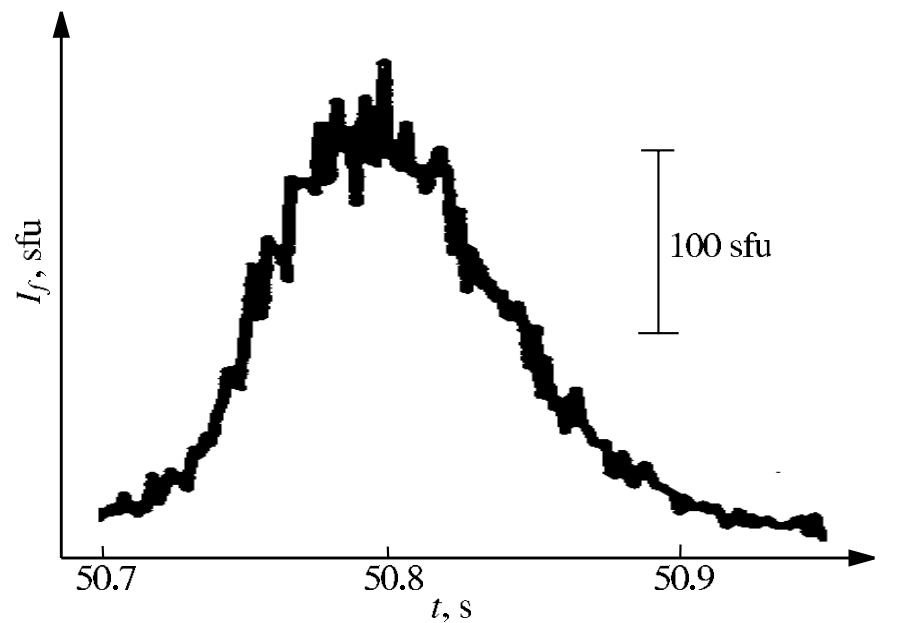

Fig. 8. Experimental time profile of radio emission intensity at the frequency $f=364 \mathrm{MHz}$. The figure is taken from (Güdel \& Benz 1990).

field with a typical scale of about the the coronal magnetic loop size virtually stabilizes the unstable (with respect of MCR) electron beams and allows them to propagate to long distances. The generation of emission becomes possible only in some local regions where a small-scale inhomogeneity of plasma density compensates the longitudinal inhomogeneity of the magnetic field and slows down the wave escape from resonance. The dependence of the emission intensity on the frequency and time in this case is the reflection of the structure and dynamics of the plasma inhomogeneities. To investigate the dependence of emission parameters on the plasma small-scale inhomogeneity, an inhomogeneity model in the form of a traveling density wave has been introduced. The main criteria for choosing the inhomogeneity model were the agreement of the calculated emission parameters with observations, and a simplification of calculations. It has been shown that, despite its simplicity, this model can account for the observed features of spectra and time profiles of spikes. By varying the parameters of the electron beam, magnetic field and plasma and their inhomogeneities and comparing the calculated and observed parameters of radio emission, one can determine the physical parameters in the sources of solar spikes, and reconstruct the dynamics of plasma inhomogeneities in particular.

Acknowledgements. The authors would like to thank Dr. A. O. Benz and Dr. D. B. Melrose for helpful comments.

This work was partially supported by the Competition Center for Fundamental Natural Sciences at St.-Petersburg University under grant No. E00-8.0-71 and by the Russian Foundation for Basic Research under grant No. 00-02-16819.

\section{References}

Aschwanden, M. J. 1990, A\&A, 237, 512

Benz, A. O. 1986, Sol. Phys., 104, 99

Csillaghy, A., \& Benz, A. O. 1993, A\&A, 274, 487

Fleishman, G. D., \& Melnikov, V. F. 1998, Uspekhi Fiz. Nauk, 168, 1265 (engl. translation: Physics-Uspekhi, 41, 1157)

Fleishman, G., \& Arzner, K. 2000, A\&A, 358, 776

Güdel, M., \& Benz, A. O. 1990, A\&A, 231, 202

Güdel, M., Aschwanden, M. J., \& Benz, A. O. 1991, A\&A, 251, 285

Le Quéau, D., Pellat, R., \& Roux, A. 1985, Ann. Geophys., 3, 273

Li, H. G. 1987, Sol. Phys., 114, 363

Melrose, D. B., \& Dulk, G. A. 1982, ApJ, 259, 844

Messmer, P., \& Benz, A. O. 2000, A\&A, 354, 287

Vlasov, V. G. 1991a, Planet. Space Sci., 39, 1223

Vlasov, V. G. 1991b, Sov. J. Plasma Phys., 17, 96

Vlasov, V. G., \& Kuznetsov, A. A. 1997, Sol. Syst. Res., 3, 207

Willes, A. J., \& Robinson, P. A. 1996, ApJ, 467, 465

Wu, C. S., Steinolfson, R. S., \& Zhou, G. C. 1986, ApJ, 309, 392

Zaitsev, V. V., \& Stepanov, A. V. 1983, Sol. Phys., 88, 297

Zarka, P., Le Quéau, D., \& Roux, A. 1986, J. Geophys. Res., 91, 13542 